\title{
Loss of HtrA2/Omi protease activity induces mitonuclear imbalance and sarcopenia via differential regulation of mitochondrial biogenesis
}

Haohan Zhou

College of Basic Medical Sciences, Jilin University

Danni Yuan

College of Basic Medical Science, Jilin University

\section{Weinan Gao}

Jilin University Second Hospital

Jiayi Tian

Jilin University First Hospital

Hongyu Sun

College of Basic Medical Sciences, Jilin University

Shuang Yu

Jilin University Second Hospital

Liankun Sun ( $\nabla$ sunlk1961@sina.com )

Jincheng Wang

Jilin University Second Hospital

\section{Research}

Keywords: a

Posted Date: March 10th, 2020

DOI: https://doi.org/10.21203/rs.2.20416/v2

License: (c) (i) This work is licensed under a Creative Commons Attribution 4.0 International License.

Read Full License 
The authors have withdrawn this preprint from Research Square 